\title{
ON A CONJECTURE OF KÀTAI CONCERNING WEAKLY COMPOSITE NUMBERS
}

\author{
JANOS GALAMBOS
}

\begin{abstract}
A number is called weakly composite if the sum of the reciprocals of its prime divisors is bounded by two. In this note it is proved that, for $n \geq n_{0}$, there is a weakly composite number between $n$ and $n+\log \log \log n$.
\end{abstract}

We call a number weakly composite if the sum of the reciprocals of its distinct prime divisors is bounded by two. We prove that, for all integers $n \geq n_{0}$, there is a weakly composite number between $n$ and $n+\log \log \log n$.

Let $q_{1}<q_{2}<\cdots<q_{N}$ be the prime divisors of $n$. We say that $n$ is weakly composite if

$$
g(n)=\sum_{j=1}^{N} \frac{1}{q_{j}} \leq 2 .
$$

One of the consequences of the result of Hausman [2] is that, for fixed $k$, there are infinitely many integers $n$ such that none of $n, n+1, n+2, \ldots, n+k$ is weakly composite. In other words, if $n_{t}, t \geq 1$, is the sequence of weakly composite numbers then, as $t \rightarrow+\infty$,

$$
\lim \sup \left(n_{t+1}-n_{t}\right)=+\infty \text {. }
$$

On the other hand, it easily follows from the most elementary results of probabilistic number theory (see, e.g., Elliott $[1$, Chapter 5, in particular the concluding remarks]) that the average of the gaps $n_{t+1}-n_{t}$ is bounded, and so (2) is very slowly diverging. When lecturing at Temple University on related topics, I. Kàtai of Budapest formulated the conjecture that the gaps $n_{t+1}-n_{t}$ must be bounded by a function of the magnitude of $\log \log \log t$. We prove this conjecture by establishing the following result.

THEOREM. For all sufficiently large real numbers $n$, there is a weakly composite number between $n$ and $n+\log \log \log n$.

ProOF. We use an idea of Kàtai [3], in which he generalizes the result of Hausman. This proof also incorporates simplifications by the referee with the referee's kind permission.

Set $k=\log \log \log n$. It suffices to show that

$$
S=\sum_{n<m \leq n+k} g(m)<2 k .
$$

Received by the editors March 28, 1985 .

1980 Mathematics Subject Classification. Primary 10A05,10H25. 
We write the left side as $S_{1}+S_{2}$, where

$$
S_{1}=\sum_{n<m \leq n+k} \sum_{\substack{p \mid m \\ p<\log n}} \frac{1}{p}
$$

and

$$
S_{2}=\sum_{n<m \leq n+k} \sum_{\substack{p \mid m \\ p \geq \log n}} \frac{1}{p}
$$

Now

$$
\begin{aligned}
S_{1} & =\sum_{p<\log n} \frac{1}{p} \sum_{\substack{n<m \leq p+k \\
p \mid m}} 1 \leq \sum_{p<\log n} \frac{1}{p}\left(\frac{k}{p}+1\right) \\
& <k \sum_{p} p^{-2}+\sum_{p<\log n} p^{-1} .
\end{aligned}
$$

From the asymptotic formula

$$
\sum_{p<x} \frac{1}{p}=\log \log x+O(1)
$$

and the elementary inequality

$$
\sum_{p} p^{-2}<\sum_{p<51} p^{-2}+\frac{1}{51}<\frac{1}{2}
$$

we see that

$$
S_{1}<3 k / 2+O(1)
$$

Next we treat $S_{2}$. Observe that if $h(m)$ is the number of prime divisors of $m$ exceeding $\log n$, then

$$
S_{2} \leq \frac{1}{\log n} \sum_{n<m \leq n+k} h(m) .
$$

For any $m \leq n+k$, we have

$$
(\log n)^{h(m)} \leq \prod_{p \mid m} p \leq n+k<2 n,
$$

from which, upon taking the logarithm, we see that

$$
h(m)<(\log 2+\log n) /(\log \log n) .
$$

Consequently, for $n$ large, $S_{2}<2 k /(\log \log n)$. Combining this with (4), we get

$$
S \leq k(3 / 2+O(1 / \log \log n)) .
$$

The inequality at (3) then follows for $n$ sufficiently large. The proof is completed.

\section{REFERENCES}

1. P. D. T. A. Elliott, Probabilistic number theory. I, Springer-Verlag, New York, 1979.

2. M. Hausman, Generalization of a theorem of Landau, Pacific J. Math. 84 (1979), 91-95.

3. I. Kàtai, A minimax theorem for additive functions, Publ. Math. Debrecen 30 (1983), 249-252.

Department of Mathematics, TU 038-16, Temple University, Philadelphia, PENNSYLVANIA 19122 